\title{
Registro de Olivancillaria vesica (Gastropoda, Olividae) sinistrógira no litoral do Estado do Paraná, Brasil
}

\author{
Marcos de Vasconcellos Gernet ${ }^{1}$, Carlos Eduardo Belz ${ }^{1}$, Carlos João Birckolz ${ }^{1} \&$ \\ Elizângela da Veiga Santos ${ }^{1,2}$
}

(1) Universidade Federal do Paraná, Laboratório de Ecologia Aplicada e Bioinvasões, Rua Rio Grande do Norte 145, Mirassol 83255-000, Pontal do Paraná, Paraná, Brasil. E-mail: lmv.gernet@gmail.com, belzoceanos@gmail.com, carlosbirc@gmail.com

(2) Universidade Federal do Paraná, Setor Litoral, Rua Jaguariaíva 512, Caiobá 83260-000, Matinhos, Paraná, Brasil. E-mail: lizveiga.ga@gmail.com

Gernet M.V., Belz C.E., Birckolz C.J. \& Santos E.V. (2018) Registro de Olivancillaria vesica (Gastropoda, Olividae) sinistrógira no litoral do Estado do Paraná, Brasil. Pesquisa e Ensino em Ciências Exatas e da Natureza, 2(2): 148-151. http://dx.doi.org/10.29215/pecen.v2i2.1068

Editor acadêmico: Silvio F. B. Lima. Recebido: 15 Outubro 2018. Aceito: 19 Novembro 2018. Publicado: 28 Dezembro 2018.

Resumo: Olivancillaria vesica (Gmelin, 1791) é um habitante comum das praias no sul e sudeste do Brasil, vivendo semi-enterrado em substratos arenosos da zona intertidal até áreas mais profundas no sublitoral. Neste trabalho, relatamos o encontro de uma concha sinistrógira de $O$. vesica, característica rara para esta espécie. Suas principais medidas são: $10.6 \mathrm{~mm}$ de altura, $5.1 \mathrm{~mm}$ de largura maior, $8.1 \mathrm{~mm}$ de altura da abertura e $2.1 \mathrm{~mm}$ de largura da abertura. O espécime foi coletado no município de Matinhos, litoral do Paraná, sul do Brasil, em cascalho biodetrítico.

Palavras chave: Caenogastropoda, Olivoidea, concha, Ilha do Farol, molusco marinho.

\section{Record of sinistral Olivancillaria vesica (Gastropoda, Olividae) in Paraná State, Brazil}

Abstract: Olivancillaria vesica (Gmelin, 1791) commonly inhabitant beaches in southern and southeastern Brazil, living semi-buried in sandy substrates from the intertidal zone to deeper areas in the sub-littoral. In this work, we report the meeting of a sinistral shell of $O$. vesica, characteristic that is rare for this species. Its main measurements are: $10.6 \mathrm{~mm}$ in height, $5.1 \mathrm{~mm}$ in width, $8.1 \mathrm{~mm}$ in height of the aperture and 2.1 $\mathrm{mm}$ in width of the aperture. It was collected in the municipality of Matinhos, coast of Paraná state, southernern Brazil, in biodetritic gravel.

Key words: Caenogastropoda, Olivoidea, shell, Farol Island, marine mollusk.

A família Olividae Latreille, 1825, é um grupo de moluscos gastrópodes que habitam fundos arenosos próximos à costa em regiões tropicais e subtropicais do mundo (Petuch \& Sargent 1986). Algumas espécies também são encontradas nas águas frias do sul da Austrália e Nova Zelândia (Smith 1998). Olivídeos são gastrópodes infaunísticos carnívoros ou detritívoros (Smith 1998). A família Olividae compreende 285 espécies (MolluscaBase 2018), das quais 30 espécies são relatadas para a costa sul-americana do Oceano Atlântico, pertencentes aos gêneros Oliva Bruguière, 1789; Ancilla Lamarck, 1799; Eburna Lamarck, 1801; Olivella Swainson, 1831; Agaronia Gray, 1839; Olivancillaria d'Orbigny, 1840; Amalda H. Adams \& A. Adams, 1853; e Jaspidella Olsson, 1956 (Absalão 2000; Absalão \& Pimenta 2003; Pastorino 2003; Rios 2009; Petuch 2013).

O gênero Olivancillaria d’Orbigny, 1840 é constituído por oito espécies [ $O$. auricularia (Lamarck, 1810); O. carcellesi Klappenbach, 1965; O. contortuplicata (Reeve, 1850); O. 
deshayesiana (Ducros de Saint Germain, 1857); O. orbignyi (Marrat, 1868); O. teaguei Klappenbach, 1964; O. urceus (Röding, 1798); e O. vesica (Gmelin, 1791)], todas com ocorrência na costa sudoeste do Oceano Atlântico (Teso \& Pastorino 2011). No entanto, Terzer \& Ryall (2005) revisaram Oliva zenopira Duclos, 1835, espécie pouco estudada e encontrada na costa sudeste do Oceano Atlântico (Angola), baseando-se em caracteres conquiliológicos, alocando-a no gênero Olivancillaria. Esse gênero é bem estudado, principalmente quanto à sistemática (Klappenbach 1964, 1965, 1966; Thomé 1966; Teso \& Pastorino 2011), e biologia das espécies (Marcus \& Marcus 1959; Jurberg 1970; Borzone 1995; Borzone \& Vargas 1999; Barreiara-Rocha 2002; Caetano \& Absalão 2002; Caetano et al. 2003; Arrighetti et al. 2012; Teso \& Giménez 2013). Tursch (1988), em uma análise bastante interessante, estudou diversos parâmetros de protoconcha de algumas espécies de Olividae, de onde veio a confirmação de diferenças supraespecíficas entre vários gêneros, incluindo Olivancillaria.

Olivancillaria vesica é um habitante comum das praias no sul e sudeste do Brasil, vivendo semi-enterrado em substratos arenosos da zona intertidal até áreas mais profundas no sublitoral (Marcus \& Marcus 1959), sendo, inclusive capturado com redes de arrasto utilizadas na pesca artesanal na plataforma continental rasa do Estado do Paraná (Belz et al. 2018). Esta espécie apresenta concha sólida, grossa, oval-oblonga, com superfície polida e brilhante. A abertura é alongada com lábio externo levemente curvado e com uma calosidade bastante discreta. Não apresenta opérculo. A columela é convexa e lisa, com dobras oblíquas anteriores e o canal sifonal é profundo, mas o sifão é curto com poucas papilas na ponta distal (Teso \& Pastorino 2011). Durante a reprodução depositam cápsulas ovígeras hemisféricas, achatadas e flexíveis. De acordo com Borzone \& Vargas (1999), espécimes vivos de Tivela mactroides (Born, 1778) e $T$. ventricosa (Gray, 1838) são os substratos de oviposição mais comuns, ao menos na região sul do Brasil. Neste trabalho relatamos o encontro de um exemplar sinistrógiro (abertura para esquerda) de $\boldsymbol{O}$. vesica, característica rara para esta espécie. Na costa brasileira, há o registro de somente duas espécies pertencentes a família Olividae com conchas sinistrógiras: Eburna lienardii (Bernardi, 1859) coletada no litoral de Recife, Pernambuco, no ano de 1996 e O. vesica (Gmelin, 1791) coletada em Arraial do Cabo, Rio de Janeiro, no ano de 2000 (Frank \& Lee 2018).

O espécime deste estudo, uma concha vazia (Figura 1), foi coletado em banco de cascalho biodetrítico, em frente à Ilha do Farol, no município de Matinhos, litoral do Paraná, Brasil (25051'02" S, 48²32'12" O), em 10 de julho de 1998, por Marcos V. Gernet. O exemplar encontra-se tombado na coleção malacológica do Museu de Zoologia da Universidade de São Paulo, lote MZSP 141102. O exemplar apresenta as seguintes medidas: $10.6 \mathrm{~mm}$ de altura, 5.1 mm de largura e abertura: 8.1 x $2.1 \mathrm{~mm}$. O espécime estudado representa um indivíduo jovem com coloração branco/creme.

Para Vermeij (1975), todos os neogastrópodes sinistrógiros foram observados em ambientes marinhos de substratos inconsolidados do tipo areia ou lama, nenhum sendo conhecido habitando substratos consolidados. Esta característica teratológica, segundo Boycott \& Diver (1923), foi determinada por herança maternal. Exemplares sinistrógiros são bastante raros sendo a dextrogiria predominante nos gastrópodes (Gould et al. 1985). De acordo com Kuroda et al. (2009), os gastrópodes sinistrógiros são recessivos e os dextrógiros são dominantes, dentro de uma determinada espécie, e a sinistrogiria é hereditária, determinada por um único locus maternal. Segundo estes autores, na espécie Lymnaea stagnalis (Linnaeus, 1758), o arranjo de blastômeros quirais no estágio de oito células determina a assimetria esquerda-direita e isto pode ser revertido alterando mecanicamente a orientação das células no embrião inicial. A abertura para esquerda em um molusco gastrópode é uma característica bastante intrigante e as explicações para este fenômeno apresentam-se apenas no campo das especulações. 


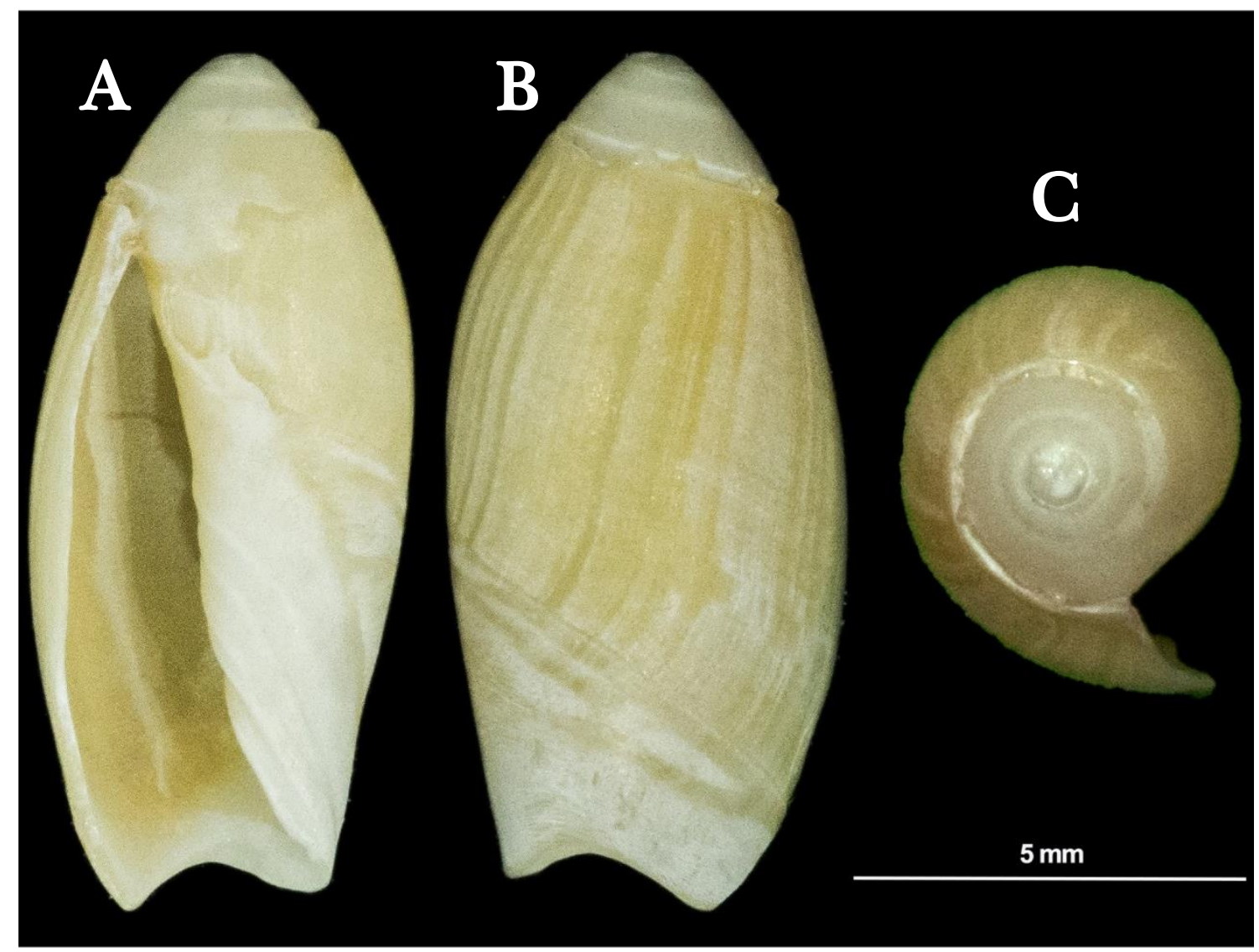

Figura 1. Concha sinistrógira de Olivancillaria vesica (MZSP 141102) coletada em banco de cascalho biodetrítico em frente à Ilha do Farol, Matinhos, litoral do Paraná, Brasil: A. Vista ventral, B. Vista dorsal, C. Vista apical.

\section{Agradecimentos}

Aos avaliadores anônimos pela revisão crítica do manuscrito.

\section{Referências}

Absalão R.S. (2000) A new species of Olivella (Neogastropoda, Olivellidae) from Brazil. Argonauta, 14(2): 11-13.

Absalão R.S. \& Pimenta A.D. (2003) A new subgenus and three new species of Brazilian deep water Olivella Swainson, 1831 (Mollusca, Gastropoda, Olivellidae) collected by the RV Marion Dufresne in 1987. Zoosystema, 25(2): 177-185.

Arrighetti F., Teso V., Brey T., Mackensen A. \& Penchaszadeh P.E. (2012) Age and Growth of Olivancillaria deshayesiana (Gastropoda: Olividae) in the Southwestern Atlantic Ocean. Malacologia, 55(1): 163-170. DOI: 10.4002/040.055.0111

Barreiara-Rocha C.A. (2002) Feeding behavior of Olivancillaria vesica auricularia (Lamarck, 1810) (Mollusca, Olividae). Thalassas, 18(2): 83-89.

Belz C.E., Ziliotto R.A., Gernet M.V., Birckolz C.J. \& Lagreze-Squella F.J. (2018) Gastropoda and Bivalvia as bycatch of the Atlantic seabob, Xiphopenaeus kroyeri, trawl fisheries in Pontal do Paraná, southern Brazil. Strombus, 24(1-2): 5-12.

Borzone C.A. (1995) Ovicápsulas de Prosobranquios (Mollusca: Gastropoda) de una playa arenosa expuesta del sur del Brasil. Iheringia, Série Zoologia, 79: 47-58.

Borzone C.A. \& Vargas K.M. (1999) Substrato para postura em Olivancillaria vesica vesica (Olividae: Mollusca) no litoral do Paraná, Brasil. Iheringia, Série Zoologia, 86: 55-60.

Boycott A.E. \& Diver C. (1923) On the inheritance of sinistrality in Limnaea peregra. Proceedings of the Royal Society of London, Series B, 95(666): 207-213. 
Caetano C.H.S. \& Absalão R.S. (2002) Imposex in Olivancillaria vesica vesica (Gmelin) (Gastropoda, Olividae) from a Southeastern Brazilian sandy beach. Revista Brasileira de Zoologia, 19(supl. 2): 215-218.

Caetano C.H.S. Veloso V.G. \& Cardoso R.S. (2003) Population biology and secondary production of Olivancillaria vesica vesica (Gmelin, 1791) (Gastropoda: Olividae) on a sandy beach in Southeastern Brazil. Journal of Molluscan Studies, 69(1): 67-73. DOI: 10.1093/mollus/69.1.67

Frank B. \& Lee H.G. (2018) Reverse Coiled Gastropods. Disponível em: https://www.jaxshells.org /reverse.htm (Acessado em 11/11/2018).

Gould S.J., Young N.D. \& Kasson B. (1985) The consequences of being different: sinistral coiling in Cerion. Evolution, 39(6): 1364-1379.

Jurberg P. (1970) Sôbre a estrutura da concha de Olivancillaria urceus (Röding, 1798) (Mollusca, Olividae). Revista Brasileira de Biologia, 30: 39-42.

Klappenbach M.A. (1964) A new species of Olivancillaria from Uruguay and Brazil. The Nautilus, 77: 132-134.

Klappenbach M.A. (1965) Consideraciones sobre el genero Olivancillaria d’Orbigny, 1840 (Moll., Gastr.) y description de dos nuevas especies de aguas argentinas y uruguayas. Comunicaciones Zoologicas del Museo de Historia Natural de Montevideo, 8: 1-10.

Klappenbach M.A. (1966) Olivancillaria vesica (Gmelin, 1791) has priority over Olivancillaria auricularia (Lamarck, 1810) (Moll., Gastr.). Archiv für Molluskenkunde, 95: 75-77.

Kuroda R., Endo B., Abe M. \& Shimizu M. (2009) Chiral blastomere arrangement dictates zygotic left-right asymmetry pathway in snails. Nature, 462: 790-795.

Marcus Ev. \& Marcus Er. (1959) Studies on Olividae. Boletim da Faculdade de Filosofia, Ciências e Letras da Universidade de São Paulo, Zoologia, 22: 99-164.

MolluscaBase (2018) Olividae Latreille, 1825. Disponível em: http://www.molluscabase.org/aphia. php?p=taxdetails\&id=23082 (Acessado em 11/11/2018).

Pastorino G. (2003) A new species of Ancillariinae (Gastropoda: Olividae) from the Southwestern Atlantic Ocean. The Nautilus, 117: 15-22.

Petuch E.J. (2013) Biogeography and Biodiversity of Western Atlantic Mollusks. New York, Boca Raton: CRC Press. 234 p.

Petuch E.J. \& Sargent D.M. (1986) Atlas of the living olive shells of the world. Charlottesville: Coastal Education \& Research Foundation. 253 p.

Rios E.C. (2009) Compendium of Brazilian Sea Shells. Rio Grande: Evangraf. 668 p.

Smith B.J. (1998) Family Olividae (p. 835-837). In: Beesley P.L., Ross G.J.B. \& Wells A. (Eds). Mollusca: The Southern Synthesis. Fauna of Australia. Volume 5. Part B. Melbourne: CSIRO Publishing. 1234 p.

Terzer G. \& Ryall P. (2005) Riscoperta e posizione sistematica di Oliva ancillarioides Reeve, 1850 ed Oliva zenopira Duclos, 1835 (Gastropoda: Olividae). Bollettino Malacologico, 41(9-12): 111-113.

Teso V. \& Giménez J. (2013) Sperm morphology of two species of Olivancillaria (Gastropoda: Olividae) from the south-western Atlantic. Journal of the Marine Biological Association of the United Kingdom, 93(3): 825-831. DOI: 10.1017/S0025315412001476

Teso V. \& Pastorino G. (2011) A revision of the genus Olivancillaria (Mollusca: Olividae) from the southwestern Atlantic. Zootaxa, 2889: 1-34.

Thomé J.W. (1966) Uma nova Olivancillaria (Gastropoda: Olividae) nas praias do Rio Grande do Sul, Brasil. Papéis Avulsos do Departamento de Zoologia, 19: 163-168.

Tursch B. (1988) Studies on Olividae. VIII. Protoconch measurements as supraspecific characters in the family Olividae. The Veliger, 31(3-4): 244-251.

Vermeij G.J. (1975) Evolution and distribution of left-handed and planispiral coiling in snails. Nature, 254: 419-420. 